\title{
Face Recognition Based on Pose-Variant Image Synthesis and Multi-level Multi-feature Fusion
}

\author{
Congcong Li, Guangda Su, Yan Shang, Yingchun Li, and Yan Xiang \\ Electronic Engineering Department, Tsinghua University, Beijing, 100084, China \\ li.congcong@gmail.com
}

\begin{abstract}
Pose variance remains a challenging problem for face recognition. In this paper, a scheme including image synthesis and recognition is proposed to improve the performance of automatic face recognition system. In the image synthesis part, a series of pose-variant images are produced based on three images respectively with front, left-profile, right-profile poses, and are added into the gallery in order to overcome the pose inconsistence between probes and images in the database. In the recognition part, a multi-level fusion method based on Gabor-combined features and gray-intensity features (GCGIF) is presented. Both amplitude features and phase features extracted through Gabor filters are utilized. Fusion is introduced in both the face representation level and the confidence level. Experiment results show that the integrated scheme achieve superior recognition performance.
\end{abstract}

Keywords: Face recognition, face synthesis, pose variance, Gabor, fusion method.

\section{Introduction}

In the past few years, techniques on face recognition have been developed a lot. Many algorithms have achieved good recognition performance in controlled conditions that faces are in frontal poses, in harmonious illumination and in neutral expression. However, there are still many open problems when face recognition technology is put into application. The face recognition vender test (FRVT) 2002 reports [1] that recognition under illumination, expression and pose variations still remains challenging. Results show that recognition rate decreases sharply when one side for matching is a face rotated to a large angle and the other is a frontal face.

Aiming to improve face recognition performance under pose variance, one way easily being thought of is to change the matching condition by adjusting face pose in one image the same with that of the other image for matching. Based on this idea, synthesizing face images in novel views is considered to be an important way and has been discussed by many researchers for a period.

In the past few years, many methods of synthesizing face image in novel views have been proposed. One common way to synthesize novel views of a face is to recover its $3 \mathrm{D}$ structure. Some current algorithms utilize a morphable $3 \mathrm{D}$ model to generate face images novel views from a single image. W. Zhao et al. [2] proposed a 
SFS (Shape from Shading)-based view synthesis technique to generate a frontal image from a profile image. It is also a single image based methods where no example based learning is carried out. These methods face to a problem in common: when only one face image is available, the texture within occluded region becomes undefined.

Vetter et al. $[3,4]$ use the linear object class approach to deal with the problem. It is a hybrid method that needs multiple images per person for training and only one image in database per person is available in recognition. It is assumed that a new face's texture can be represented as a linear combination of the texture from a group of example faces in the same view, and the combination coefficients can be used to synthesize the face image in another view. However, another difficulty appears. Since the generated texture is a linear combination of the textures in training database, some individual characteristic information would be lost, such as little scar, beauty spot, and so on.

Besides the two categories of methods, another category of methods are multiple image based methods where multiple images of variant poses per person are available. To collect multiple images per person in the database directly by capturing is hardly carried out; however, with synthesis technique, we can generate multiple images through only one or a limited quantity of images. It is the method category that this paper adopted.

The proposed work in this paper mainly aims to be applied to criminal identification. In the original criminal database, there are commonly three face images of every person who has a criminal history, respectively in the frontal pose, left profile pose and right profile pose. In our work, we utilize these three images to synthesize more images of the same person with different rotation angles. The synthesized images are then put into the database to increase the pose variety. In the recognition stage, when a probe comes, it is first estimated about its pose, and then compared with a sub-database selected according to the principle that images in the sub-database has the most similar pose with that of the probe.

The synthesis work is carried on based on Stereopsis and projective geometry. Before synthesis, ASM algorithm is improved to ensure the accuracy of face alignment. 3D shape reconstruction and triangle-based cubic spline interpolation are introduced for generating the shape in new pose. Multi-resolution spline technique is adopted in texture synthesis. Proper integration of all these techniques results in satisfying synthesis performance which retains major characteristics of a face.

Besides the synthesis work, this paper also studies the area of face recognition algorithm. Although the synthesis work has helped to adjust the pose of images in the database similar to that of the probe, another problem is left. That is, how to make the recognition algorithm robust for two profile image matching. Therefore, in this work, we tried to introduce Gabor wavelet feature since Gabor features have the properties of orientation selectivity, spatial frequency selectivity, and insensitivity in positioning error. At the same time, gray-intensity feature was discarded; instead, it was combined with Gabor features at different stage by trials. Finally, we present a twolevel fusion algorithm for recognition based on Gabor-amplitude features, Gaborphase features and Gray-Intensity features. This method combines the Gabor amplitude features and phase features in the PCA level to form Gabor-combined features, which are then joined with gray intensity features in the confidence level to form the final classifier. 
Combining the synthesis work and the recognition work, this paper makes contributions in mainly three aspects:

1. It generates multiple images in variant poses from three images per person and makes the images on sides for recognition have similar pose so that the recognition performance is improved.

2. Since the synthesis is based on more than one image, the texture contained in the three original images can cover all area of the face so that individual details are retain;

3. Since the synthesis is carried on aforehand before recognition, it brings no time problem for recognition, and thus the proposed scheme is suitable for real-time face identification applications.

The rest of the paper is organized as follows: Section 2 provides an overview of the whole proposed scheme; Section 3 introduces major techniques in the proposed image synthesis course including face alignment, shape reconstruction, texture synthesis and pose estimation. Section 4 demonstrates the framework and the detail of the proposed two-level fusion recognition method based on multiple image features. Experiment results are given in Section 6, and this paper is concluded in section 7.

\section{Overview of the Proposed Scheme}

Aiming to solve the problem of recognizing an image with face pose in a large rotation angle, an integrated scheme is designed, which consists of two parts: database image synthesis part and face image recognition part. As shown in Fig.1, before recognition, synthesis work would be done based on three categories of databases, respectively with frontal poses, left-profile poses and right-profile poses. Through synthesis, the database will be enlarged by adding images with other poses. In therecognition stage, when a probe comes, the proposed system will first estimate its face pose and then pick out a sub-database whose images have the most similar pose with that of the probe. So the recognition work will carried on between the probe and

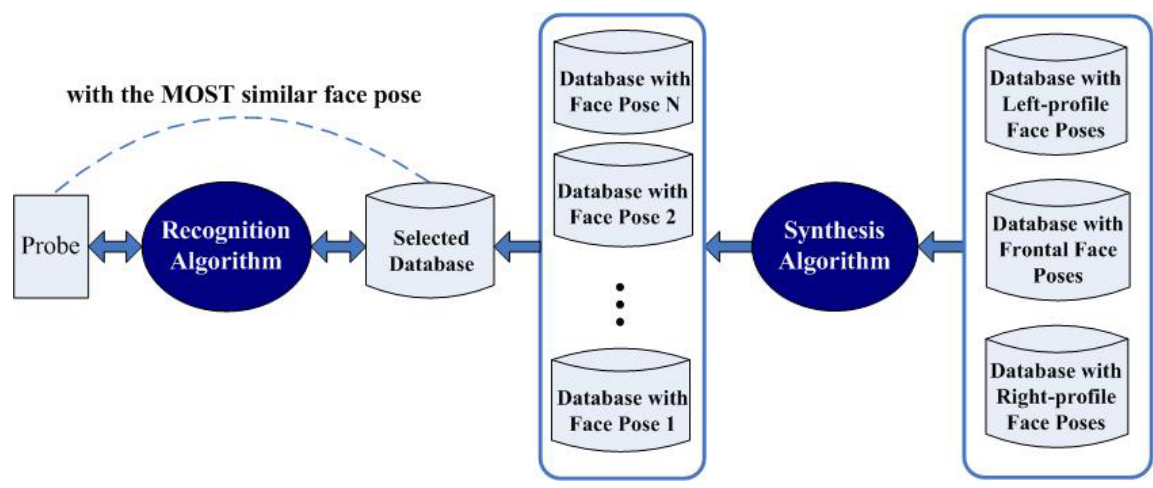

Fig. 1. Overview of the whole scheme 
the selected sub-database. Therefore, the most important work in this scheme is the synthesis stage and the recognition stage, both of which have crucial affect on the final recognition performance.

\section{Pose-Variant Face Image Synthesis}

This section gives an overview of the synthesis proposed in our scheme and introduces briefly the key techniques utilized for synthesis.

\subsection{Synthesis Framework}

Frontal, left-side and right-side face images are utilized together to produce images with any possible pose. These synthesized images then compose sub-datasets of the gallery, each of which represents a pose type. Fig.2 illustrates the whole synthesis process. Face alignment, new shape generation and new texture synthesis are crucial parts in the synthesis stage.

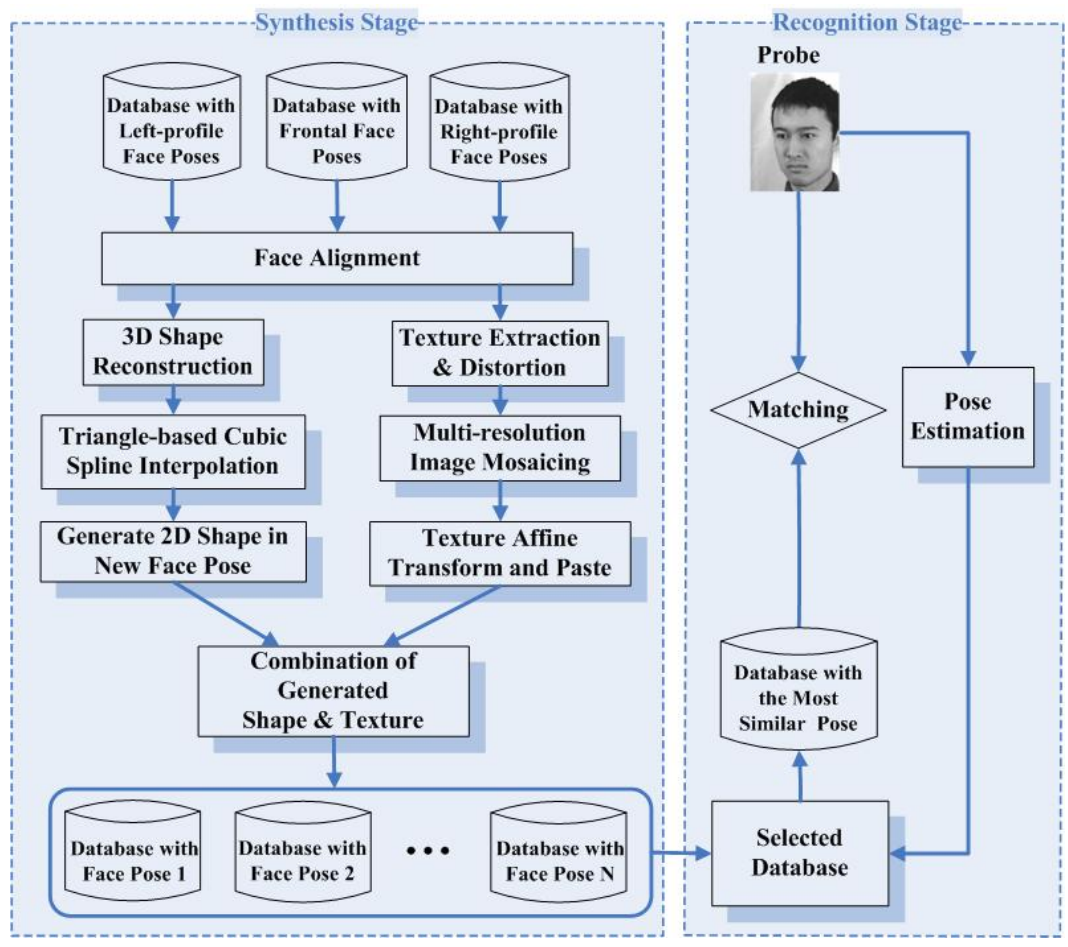

Fig. 2. Framework of the synthesis stage

\subsection{Face Alignment}

An improved ASM (Active Shape Models) method is chosen to extract the face feature points in this paper. It is hard for the conventional ASM to get accurate result 
on each feature point; what's more, the performance depends heavily on the initial positions of the landmarks. According to the structure of face, the edge information and part information of face are introduced to the matching process of ASM, which improved the performance of ASM [5].

The face images are firstly normalized. Then the improved ASM algorithm help to extract 105 feature points to represent the front face shape and 57 feature points to represent the profile face shape respectively, as shown in Fig.3.
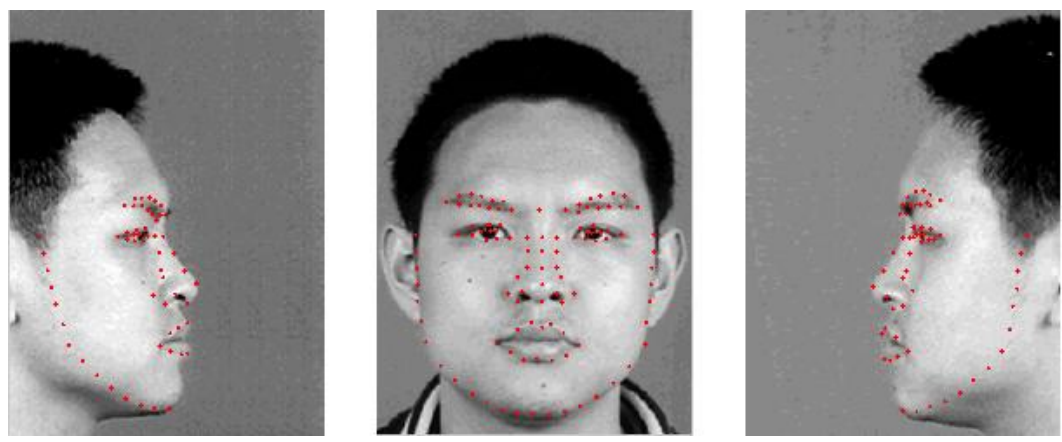

Fig. 3. Feature points extracted by the improved ASM algorithm

To ensure the corresponding relation between feature points in different images, feature points of some face parts like contour are connected and fitted by polynomial curve. As shown in Fig. 4, half of the contour is fitted at two stages. At the first stage shown in the left, feature points are roughly fitted and the point parallel to the corner of mouth is considered to be a subsection point. Then the contour is fitted separated by two curves as shown in the right. Then the contour feature points are adjusted on the curves in well-proportioned distribution. Besides fitting the contour, similar operations are also carried onto the other facial parts. The alignment of the face feature points provides an important basis for shape reconstruction of frontal face, which would be mentioned in the next section.

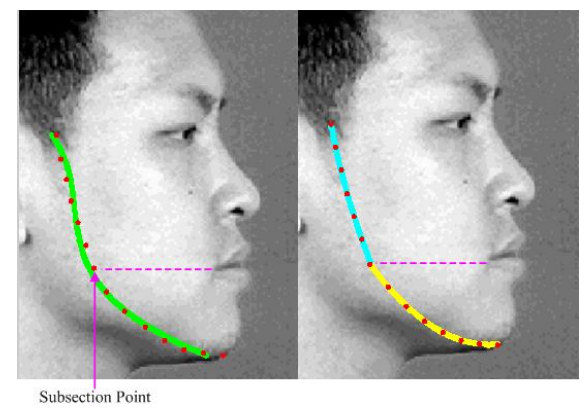

Fig. 4. A two-stage fitting to the contour with polynomial curves 


\subsection{Shape Reconstruction}

One face image includes shape information and pure texture image. So a face image can be separated into shape information and texture information. If we have these two kinds of information, we can form a new face image.

The 3D shape of a given face is reconstructed based on the aligned feature points extracted from the three source images. For each 3D point, its $x$ and $y$ coordinate values can be obtained from the frontal image and its $\mathrm{z}$ coordinate value can be obtained from either profile image, as shown in the Fig. 5 below.
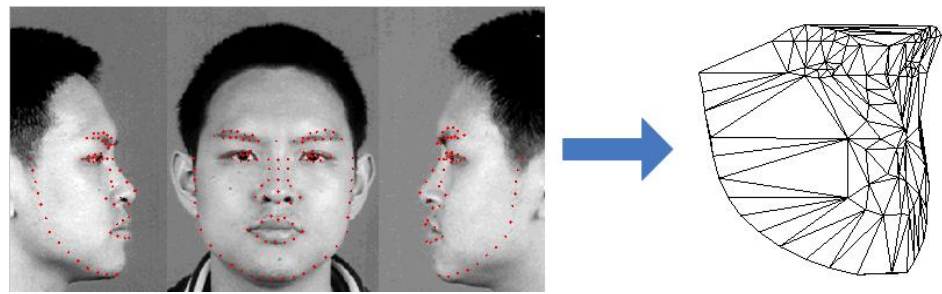

Fig. 5. Demonstration of 3D shape reconstruction based on stereoscopy

The 3D shape formed directly from the source images contains only 105 points, which are not enough for representing the face shape. So based on these sparse feature points, we introduce the triangle-based cubic spline interpolation technique [6][7][8] to generate dense regularly spaced grid, as shown in Fig.6.

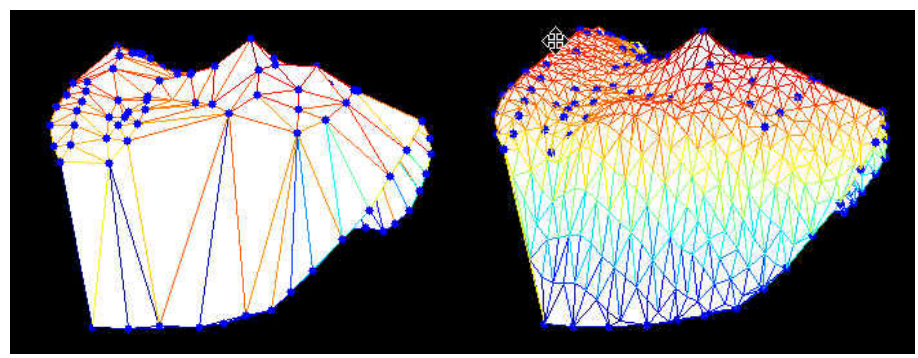

Fig. 6. Dense regularly spaced grid generation for representing face shape

With the dense grid, we can generate the 2D face shape in new pose based on stereoscopy. Since some parts of the face would be occluded in the new pose, we need to compute the new edges of all face parts, especially the nose part and the contour part.

\subsection{Texture Synthesis}

As mentioned in Section. 1, one advantages of the proposed method in this paper is that there is enough information for texture synthesis. To make full use of the textures 


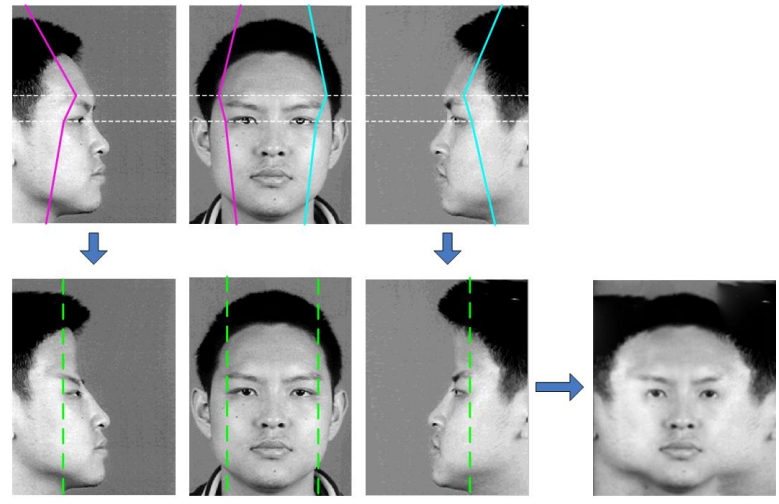

Fig. 7. Image transform and multi-resolution mosaic

of three source images, transform is carried on according to a set of principles as shown in Fig.7. Based on the curves marked in Fig.7, images in the first row is transformed to those in the second row, which are then cut and combined with the help of multi-resolution spline technique [9] to generate the mosaic image in rightdown corner.

In order to generate the image in new pose, the new $2 \mathrm{D}$ shape points and their corresponding positions in the mosaic image are needed. Since in 3.4 we have already gained the new 2D shape points, we can also compute out their corresponding positions in the mosaic images by utilizing the relationship between $3 \mathrm{D}$ coordinates and $2 \mathrm{D}$ coordinates and all the parameters in the image transform and mosaic course.

After the corresponding points are confirm, Delaunay triangularization following the same principle is introduced to form multiple triangles to connect the points. Then the triangle based affine transform is used to span the selected part of the mosaic face image to fit for the destination shape. Equation (1) describes this affine transform process.

$$
\left[\begin{array}{l}
x^{\prime} \\
y^{\prime}
\end{array}\right]=\left[\begin{array}{ll}
a & b \\
c & d
\end{array}\right]\left[\begin{array}{l}
x \\
y
\end{array}\right]+\left[\begin{array}{l}
O_{x} \\
O_{y}
\end{array}\right]
$$

Fig. 8 is an example of the affine transform for texture synthesis.
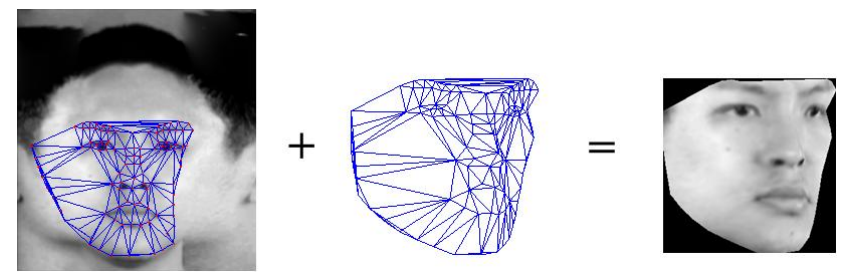

Fig. 8. Process of affine transform for texture synthesis 


\subsection{Pose Estimation}

As shown in Fig.2, in order to select the sub-database whose images have the similar pose as the probe, the probe's probe should be estimated. In this work, we require the input probe with a rotation angle no larger than $45^{\circ}$. The probe is first preprocessed such as feature-positioned and normalized. It is rectified and normalized geometrically according to the auto located key-point positions. In the geometric normalization step, not only the eyes but also the chin middle point would be automatically located. Then each face image would be scaled and rotated so that the eyes are positioned in line and the distance between the chin point and the center of the eyes equals a predefined length. After that, the face image is cropped to a given size. The examples of training images in TH database (built by ourselves) are shown in Fig.9.
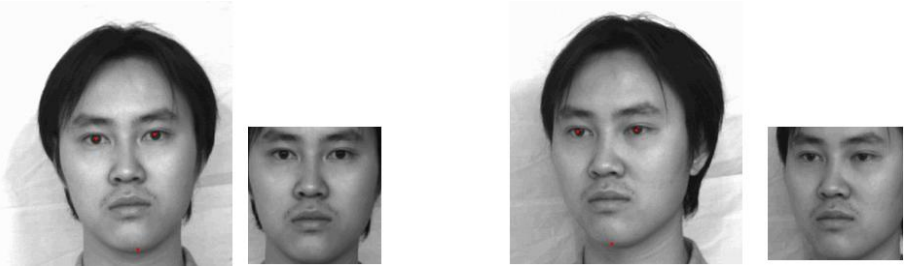

Fig. 9. The training face images in $\mathrm{TH}$ database

It is essential to extract features from images utilizing the composite PCA (principle component analysis) and projecting face images to the eigenspace. Given a set of samples $X_{i} \in \mathbb{R}^{N}$ represented face images by column vectors. The transformation matrix can be formed by using eigenvectors which normalized to unit matrix $T$.The projection of $X_{i}$ into the $\mathrm{N}$-dimensional subspace can be expressed as

$$
\alpha=\left\{\alpha_{1}, \cdots, \alpha_{N}\right\}=X_{i}^{T} \cdot T
$$

The shape feature is shown in Fig.10 in next page. The feature points can give geometric characteristic. $A B$ and $A^{\prime} B^{\prime}$ are the distance between two eyes when pose angle is 0 and $\beta$ degree respectively. Set radius as 1 .

$$
A^{\prime} E^{\prime}=A^{\prime} B^{\prime}=\sin (\theta+\beta)+\sin (\theta-\beta)=2 \sin \theta \cos \beta
$$

Since distance $A B=2 \sin \theta$, then pose angle

$$
\beta=\arccos \left(\frac{A^{\prime} E^{\prime}}{A B}\right)
$$

Set weights of two weight parameters $\alpha$ and $\beta$ after two groups of features are gained. The new eigenvector $\xi$ is

$$
\xi=p \cdot \alpha+q \cdot \beta, \text { where } p+q=1
$$




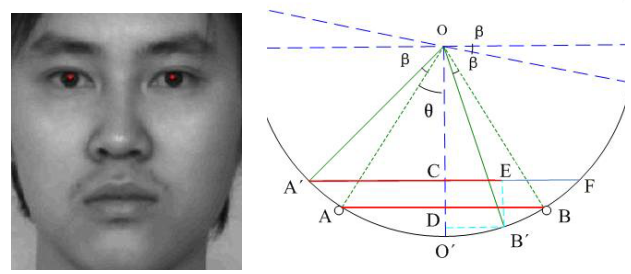

Fig. 10. Shape feature points and the configuration of pose variance

SVM (support vector machine) is to find the optimal linear hyperplane which the expected classification error for unseen test samples is minimized [10]. According to the structural risk minimization principle, a function that classifies the training data accurately will generalize best regardless of the dimensionality of the input space.

Each training sample $x_{i}$ is associated with coefficient $a_{i}$. Those samples whose coefficient $a_{i}$ is nonzero are Support Vectors (SV) of the optimal hyperplane. $f(x)$ is an optimal SVM classified function. $y_{i} \in(+1,-1)$.

$$
f(x)=\sum_{\text {vector }} y_{i} a_{i} K\left(x_{i}, x\right)+b
$$

where $K$ is a kernel function. Here we use linear kernel, $\phi\left(x_{i}\right)=x_{i}$, then $K\left(x_{i}, x_{j}\right)=x_{i} \cdot x_{j}=x_{i}^{T} x_{j}$.

The PCA projection values of samples to eigenspace were as SVM input parameters and the optimal hyperplane that correctly separates data points were found. Combining the PCA and SVM classifier, we can draw better classification results. Then the pose angle will be acquired.

\section{Multi-level Multi-feature Fusion Method}

This section introduces the recognition algorithm proposed in our work, which is a two-level fusion method based on three types of image features.

\subsection{Fusion Framework}

Three types of face representations are introduced in this method, including gray-level intensity features, Gabor amplitude features and Gabor phase features. Fig.11 illustrates the process of feature extraction and the design of two-level fusion. In the PCA level, shown in the left gray frame, we make a fusion between Gabor amplitude features and phase features. These two features are then treated as a whole in the later steps. Another fusion is introduced in the confidence level between the matching result based on Gabor-combined features and that based on gray intensity features, as shown in the right gray frame. This two-level fusion method based on Gaborcombined features and gray-intensity features is called GCGIF method in the subsequent part of this paper. 


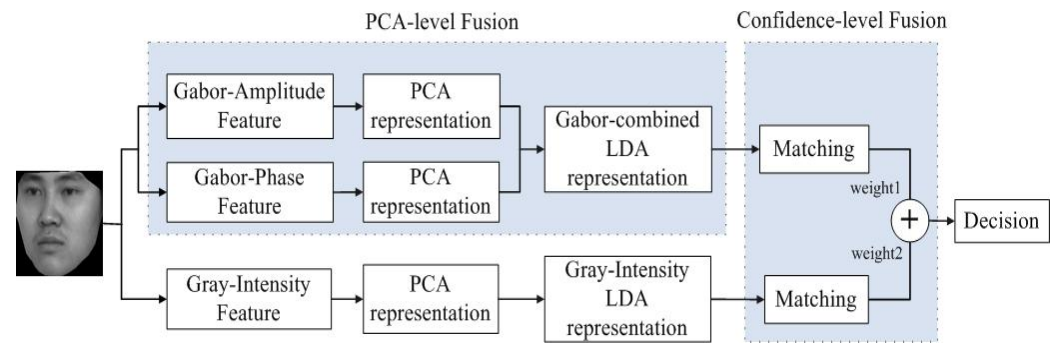

Fig. 11. Design of the proposed method GCGIF

\subsection{Feature Extraction}

Extracting Gray-level Intensity Feature: Face images are rectified and normalized geometrically according to the auto located key-point positions, as mentioned in Section 3.5. After these processes, the face image is cropped to a given size $(90 * 120)$. To reduce the affection of different hairstyles and backgrounds, a mask is put on the face image. Moreover, histogram equalization is introduced to decrease the influence from light and complexion variation. The preprocessing procedure is shown in Fig.12 (a).

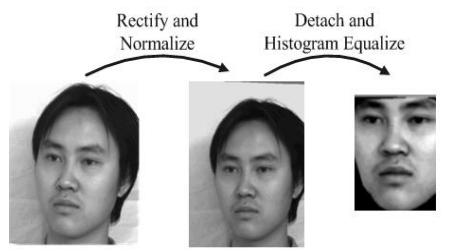

(a)

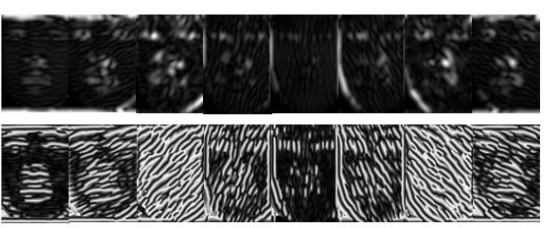

(b)

Fig. 12. (a) formation of gray intensity feature; (b) examples of Gabor amplitude features and phase features

Extracting Gabor Features: Gabor wavelet has been introduced to image analysis due to its biological relevance and its ability in representing spatial locality and orientation. In our experiments, we consider both Gabor amplitude and phase for face image representation.

The 2D Gabor wavelet components can be defined as equations (7) and (8):

$$
\begin{array}{r}
G_{\vec{k},+}(\vec{r})=k^{2} \exp \left(-k^{2}\left\|\vec{r}-\vec{r}_{0}\right\| / 2 \delta^{2}\right)\left\{\cos \left[\vec{k}\left(\vec{r}-\vec{r}_{0}\right)\right]-\exp \left(-\delta^{2} / 2\right)\right\} / \delta^{2} \\
G_{\vec{k},-}(\vec{r})=k^{2} \exp \left(-k^{2}\left\|\vec{r}-\vec{r}_{0}\right\|^{2} / 2 \delta^{2}\right) \sin \left[\vec{k}\left(\vec{r}-\vec{r}_{0}\right)\right] / \delta^{2}
\end{array}
$$

where $\vec{k}=k \exp \left(j \theta_{v}\right), k=k_{\max } / \lambda^{u}, \theta_{v}=\pi v / n$.

Here $\vec{k}$ is the filter wave-vector, determines the spatial frequency and orientation tuning of the filter. If $n=8, u \in\{0,1,2,3,4\}, v \in\{0,1, \cdots, 7\}, \delta=\pi, k_{\max }=\pi / 2$, and $\lambda=2$, a family of Gabor filters with 5 scales and 8 orientations are generated. 
When a gray-intensity image is convolved with these Gabor filters, responses of the filters would be combined into a vector, with components given below:

$$
R_{\vec{k}, \pm}\left(\vec{r}_{0}\right)=\int G_{\vec{k}, \pm}\left(\vec{r}_{0}, \vec{r}\right) I(\vec{r}) d \vec{r}
$$

where $I$ represents the input gray intensity image.

Then Gabor amplitude features and phase features of the corresponding face image could be computed as follows:

$$
R_{\vec{k}}=\sqrt{R_{\vec{k},+}^{2}+R_{\vec{k},-}^{2}}, \quad \psi_{\vec{k}}=\arctan \left(R_{\vec{k},-} / R_{\vec{k},+}\right)
$$

Fig.12(b) gives examples of outputs from Gabor filters, with amplitude features in the first row and the corresponding phase features in the second row, both are at the condition of $u=2$.

The Gabor wavelet transform of an image is the collection of the coefficients of all the pixels $(90 * 120)$. To reduce the dimensionality, the pixels are sampled to $24 * 32$. To further reduce the redundancy of features, PCA (Principle Component Analysis)[11] and LDA (Linear Discriminant Analysis)[12] are employed.

\subsection{Selection of Fusion Mechanism}

We study fusion mechanism at both the representation level and the confidence level, as shown in Fig.13 and Fig.14.

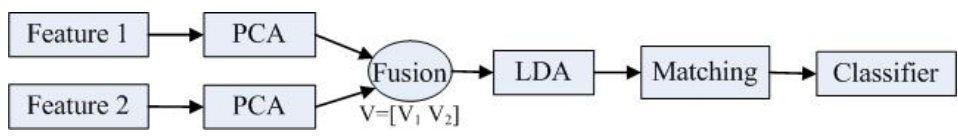

Fig. 13. Feature fusion at the PCA level

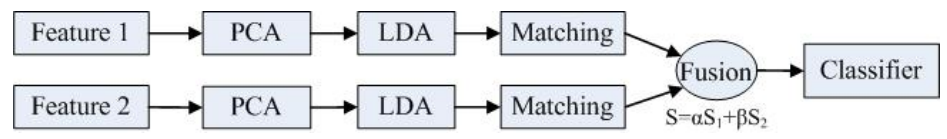

Fig. 14. Feature fusion in the confidence level

At the representation level, vectors extracted by PCA from different kinds of features are combined firstly. Then the combined vector enters LDA. At last, the feature vector obtained by LDA is the final representation for the face image and would be used for matching before the final decision.

The mechanism of confidence-level fusion works as shown in Fig.14. Different features are first processed by PCA and LDA and the LDA results are used for matching respectively. Then different feature-matching results are calculated into final similar-scores based on the weighted sum rule.

Both the above fusion mechanisms may help improve the recognition task. Which mechanism should be utilized is decided through experiment trials here. 
Table 1. Recognition performance without fusion

\begin{tabular}{cccc}
\hline Without Fusion & $\begin{array}{c}\text { Gabor-Amplitude } \\
\text { PCA-LDA }\end{array}$ & $\begin{array}{c}\text { Gabor-Phase PCA- } \\
\text { LDA }\end{array}$ & $\begin{array}{c}\text { Gray-Intensity } \\
\text { PCA-LDA }\end{array}$ \\
\hline Recognition Rate & $70.0 \%$ & $63.8 \%$ & $67.8 \%$ \\
\hline
\end{tabular}

Table 2. Recognition performance with different fusion manners

\begin{tabular}{|c|c|c|c|}
\hline \multicolumn{2}{|c|}{ Features for Fusion } & \multirow{2}{*}{ Fusion Level } & \multirow{2}{*}{ Recognition Rate } \\
\hline Feature1 & Feature 2 & & \\
\hline Gabor-Amplitude & Gabor-Phase & PCA & $74.5 \%$ \\
\hline Gabor- Amplitude & Gabor-Phase & $\begin{array}{l}\text { Confidence } \\
(\alpha: \beta=5: 2)\end{array}$ & $72.3 \%$ \\
\hline Gabor-Combined & Gray-Intensity & PCA & $78.4 \%$ \\
\hline Gabor-Combined & Gray-Intensity & $\begin{array}{l}\text { Confidence } \\
(\alpha: \beta=3: 2)\end{array}$ & $81.9 \%$ \\
\hline
\end{tabular}

Face recognition performance is evaluated based on part of the TH-database [13]. The gallery consists of images of 160 people with one frontal image per person. The probe dataset consists of 320 images whose left-right rotation angle is either -15 or +15 degree. The recognition rate means the fraction of probes that have rank top fifth in the identification task. Table. 1 shows the recognition performance based on each type of features without fusion.

Table. 2 compares the performance of different fusion mechanisms. Comparing with Table.1, it is obvious that fusions of features do help to improve the recognition performance. Due to greater correlation between Gabor amplitude and phase features, we first consider making fusion between them. The upper part of Table. 2 shows that combing Gabor amplitude and phase features in PCA level outgoes that in confidence level, so the former is adopted to form the Gabor-combined features. Then the nether part of Table. 2 shows that further fusion between Gabor-combined feature and grayintensity feature in the confidence level is better and should be adopted. The reason behind the results may be that features with more inherent relativity, just like amplitude feature and phase feature both extracted by Gabor filters, adapts to fusion at an earlier stage.

According to results in Table.2, the fusion mechanism for GCGIF algorithm was formed as Fig.11 illuminated. Several classic algorithms are also evaluated in our experiments, including the PCA algorithm, the combined PCA and LDA algorithm (PCA+LDA) --both based on gray intensity features-- and the PCA+LDA algorithm based on Gabor amplitude features (G PCA+LDA). A previously released fusion algorithm MMP-PCA (Multi-Modal Part face recognition method based on Principal Component Analysis) [14], is also tested for reference. Table.3 shows that GCGIF delivers the best result in the above mentioned experiment condition.

Table 3. Recognition performance of different methods

\begin{tabular}{ccccc}
\hline Methods & PCA-LDA & G PCA-LDA & MMP-PCA & GCGIF \\
\hline Recognition Rate & $67.8 \%$ & $70.0 \%$ & $76.3 \%$ & $\mathbf{8 1 . 9 \%}$ \\
\hline
\end{tabular}




\section{Experiment Results}

In this section we describe our experiments on the TH face database. The $\mathrm{TH}$ face database contains face images of 160 persons with left-right rotation angles from -90 degree to +90 degree in the interval of 15 degree. We select the frontal, left-profile, right-profile images as original gallery and select images from -45 degree to 45 degree in the interval of 15 degree to form probe sets. Images in every probe set have the same face pose.

In our experiment, the frontal, left-profile, right-profile images are first utilized to synthesize multiple face images with left-right rotation angles from -45 degree to +45 degree in interval of 5 degree, through the proposed synthesis framework introduced in Section.3. The synthesized images then constitute multiple sub-databases, each of which represents an individual pose.

Fig.15 gives two examples of face synthesis based on three images. For each person, the first row are original images with frontal, left-profile and right-profile poses; the following two rows are the synthetic face images with poses from -45 degree to 45 degree at an interval of 5 degree.
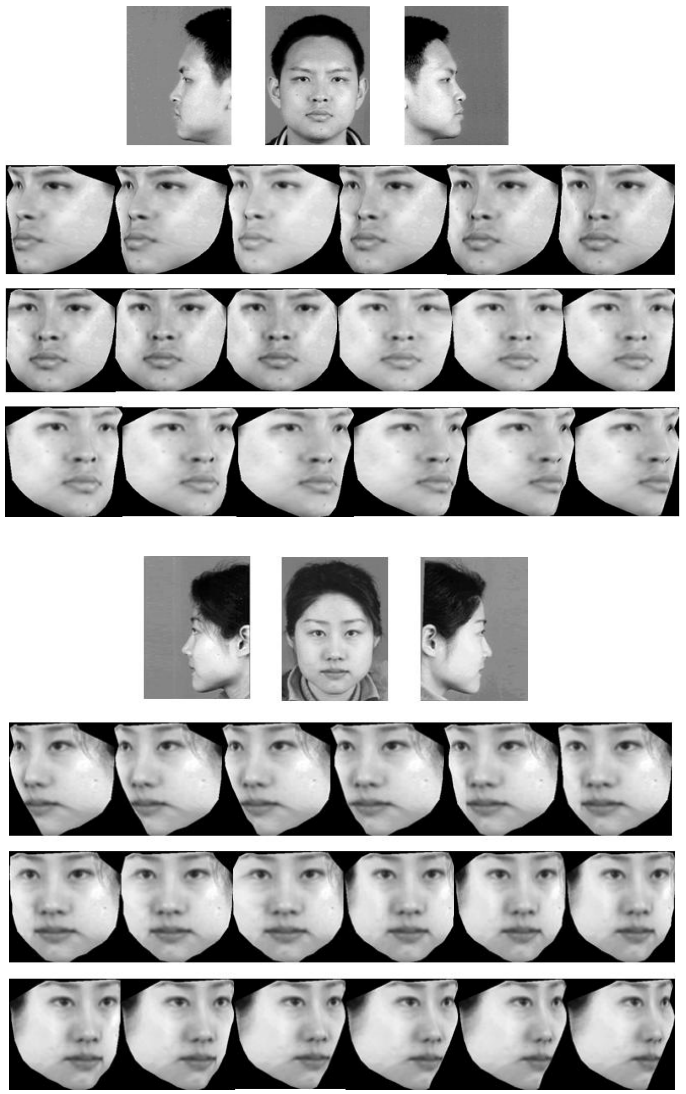

Fig. 15. Examples of the face synthesis results 
The recognition experiments are based on an identification task. Recognition rate is tested on each probe set. The recognition rate means the fraction of probes that have rank top fifth in the identification task. The recognition algorithm adopted the GCGIF algorithm presented in Section 5. To better evaluate the proposed scheme in this paper, we compare the recognition performances in database with synthesized images and in database without synthesized image. In both the situations, the gallery consists of images of 160 people and each probe set contains 160 face images. The experiment results are shown in Table. 4.

Table 4. Recognition performance in the original gallery and that in the synthetic gallery

\begin{tabular}{ccc}
\hline $\begin{array}{c}\text { Probe } \\
\text { (Pose) }\end{array}$ & $\begin{array}{c}\text { Recognition rate in the database } \\
\text { containing only frontal, left- } \\
\text { profile, right-profile images }\end{array}$ & $\begin{array}{c}\text { Recognition rate in the database } \\
\text { containing frontal, left-profile, } \\
\text { right-profile images and also } \\
\text { synthesized images }\end{array}$ \\
\hline L 15 & $81.3 \%$ & $\mathbf{8 5 . 0 \%}$ \\
L 30 & $58.1 \%$ & $\mathbf{7 5 . 6 \%}$ \\
L 45 & $29.4 \%$ & $\mathbf{5 4 . 4 \%}$ \\
R 15 & $81.9 \%$ & $\mathbf{8 6 . 3 \%}$ \\
R 30 & $56.9 \%$ & $\mathbf{7 5 . 0 \%}$ \\
R 45 & $25.6 \%$ & $\mathbf{5 5 . 6 \%}$ \\
\hline
\end{tabular}

Table. 4 shows the recognition performance of different probe sets. In Table.4, "L $\mathrm{xx}$ " means the images in the set has a face pose with $\mathrm{xx}$ degree's rotation to its left. Similarly, "R" means "Right". From Table.4, we can see that the recognition rates in the third column are much higher than the second column in the same row, which indicates that the synthesis of pose-variant images for the database did help to improve the recognition performance significantly.

\section{Conclusions}

In this paper, we design an integrated scheme for pose-variant face recognition. There are mainly two contributions.

First, Synthesis is introduced to produce a series of pose-variant face images based on three images with frontal, left-profile and right-profile poses respectively in order to overcome the difficulty of image matching between faces with inconsistent poses. The generated images are then added to the gallery to increase the pose variety of each individual before the recognition work. In the recognition stage, when a probe comes, it will first be estimated about its pose and then be compared with images in a sub-dataset with the most similar pose. Since the synthesis is processed before the recognition work, the recognition speed has not been affected. Although a small quantity of unavoidable estimation and alignment errors may affect the final reconstruction accuracy, experiment results show that most of the information important for recognition has been retained and helps to improve the recognition performance. 
Secondly, a two-level fusion algorithm for face recognition based on GaborCombined features and Gray-Intensity features, GCGIF, is presented in order to improve the recognition performance. This method combines the Gabor amplitude features and phase features in the PCA level to form Gabor-combined features, which are then joined with gray intensity features in the confidence level. This two-level fusion mechanism is selected through experimental trials and rational conjecture on correlation between features. Experimental results show that GCGIF achieves superior performance.

Due to these two contributions, experiments show that the proposed scheme achieves great improvement for pose-variant face recognition. Without expensive time cost, this method is suitable for real-time identification applications.

\section{References}

1. Phillips, P.J., Grother, P., Ross, J., Blackburn, D., Tabassi, E., Bone, M.: Face Recognition Vendor Test 2002: Evaluation Report (March 2003)

2. Zhao, W., Chellappa, R.: SFS based view synthesis for robust face recognition. Int. C. Automatic Face and Gesture Recognition, 285-292 (2000)

3. Vetter, T.: Synthesis of novel views from a single face image. International Journal of Computer Vision 28(2), 103-116 (1998)

4. Vetter, T., Poggio, T.: Linear object classes and image synthesis from a single example image. IEEE Transactions on Pattern Analysis and Machine Intelligence 19(7), 733-742 (1997)

5. Du, C., Su, G.D., Lin, X.G., Gu, H.: An Improved Multi-resolution Active Shape Model for Face Alignment. Jounal of Optoelectronics Laser 15(12), 706-710 (in Chinese, 2004)

6. Barber, C.B., Dobkin, D.P., Huhdanpaa, H.T.: The Quickhull Algorithm for Convex Hulls. ACM Transactions on Mathematical Software 22(4), 469-483 (1996)

7. National Science and Technology Research Center for Computation and Visualization of Geometric Structures (The Geometry Center), University of Minnesota (1993)

8. Watson, D.E.: Contouring: A Guide to the Analysis and Display of Spatial Data. Pergamon, Tarrytown, NY (Elsevier Science, Inc.) (1992)

9. Burt, P.J., Andelson, E.H.: A Multiresolution Spline With Application to Image Mosaics. ACM Transactions on Graphics 2(4), 217-236 (1983)

10. Vapnik, V.: Statistical Learning Theory. John Wiley \& Sons, New York (1998)

11. Turk, M., Pentland, A.: Eigenfaces for Recognition. Journal of Cognitive Neuroscience 3, 72-86 (1991)

12. Martinez, A.M., Kak, A.C.: PCA versus LDA. IEEE Trans. on PAMI 23(2), 228-233 (2001)

13. Li, C.C., Su, G.D., Meng, K., Zhou, J.: Technology Evaluations on TH-FACE Recognition System. In: Zhang, D., Jain, A.K. (eds.) Advances in Biometrics. LNCS, vol. 3832, pp. 589-597. Springer, Heidelberg (2005)

14. Su, G.D., Zhang, C.P., Ding, R., Du, C.: MMP-PCA face recognition method. Electronics Letters 38(25) (2002) 